\title{
Improved Models for Precession and Nutation
}

\author{
P. M. Mathews \\ Department of Theoretical Physics, University of Madras, Chennai \\ 600025, India
}

\begin{abstract}
The modeling of nutations and precession has advanced to the point where the rms of residuals between theory and the observational estimates from the VLBI data of the past decade is only 0.16 mas in $\Delta \psi \sin \epsilon_{0}$ as well as in $\Delta \epsilon$. Such a fit is provided by the MHB2000 nutation series (Mathews et al., 2000) based on geophysical theory with a few basic Earth parameters estimated by a fit to nutation-precession data, and its accompanying precession rate. A brief account of the series is presented, along with an outline of the theoretical background and of the geophysical information of interest obtained in the process of constructing the series. A series due to Shirai and Fukushima (2000) also gives a somewhat comparable fit to data, improving on the IERS 1996 series, but it is essentially empirical and provides no geophysical insights.
\end{abstract}

\section{Introduction}

This paper will focus on recent developments in the geophysical modeling of precession and nutation which have made it possible to account for observations of these phenomena at a level of accuracy that is close to the precision of the observational data. The survey presented by Veronique Dehant at this Colloquium may be referred to for an overview of developments in various aspects of the modeling process and various approaches to modeling.

One of the approaches, employed first by T. A. Herring in his construction of the IERS 1996 nutation series for forced nutations (McCarthy, 1996), is based on estimation of four of the complex parameters in the resonance formula (6) below, by a direct fit to nutation time series derived from VLBI data. Improvements over the already high accuracy of IERS 1996 have been effected recently by Shirai and Fukushima (2000) through refinements of the method and the use of more extensive data, in their work reported at this meeting. The values obtained for the resonance parameters by this approach may not, however, be interpreted in geophysical terms, because sum rules relating these parameters, which follow from physical theory, are not enforced in the process of fitting to data. In this sense, this approach may be termed "empirical".

The second type of approach makes direct use of geophysical theory, but through a variety of methodologies. The detailed modeling of the Earth's interior structure that is called for by the method of Wahr (1981) and Dehant and Defraigne (1997) is not complete enough yet to account for nutations at a level approaching the precision of current observational data. Major advances have 
been made possible, however, by the use of an analytic formulation of nutation theory. In this type of theory, employed by Mathews et al..(2000), the nature of the dependence of the nutation amplitude on the basic Earth parameters (BEP) that influence nutation is known in analytic terms. This knowledge is exploited in the estimation of the most influential of these parameters through a fit of the expression for the nutation amplitude to observational data. Once the estimation is done, knowledge of the functional dependence of the nutation amplitude on the BEP makes it possible to compute the amplitude of the nutation for any frequency. The remainder of this paper will be devoted to a presentation of the new nutation series MHB2000 and of the geophysical theory on which it is based. Though the paper of Getino and Ferrándiz presented at this Colloquium claims an accuracy at least as good as that of MHB2000, we refrain from attempting to discuss that work for want of concrete information about the specifics of the Hamiltonian used by them and the parameters involved, and about the parameters adjusted in their fit to the IERS1966 series (not the observational data).

In constructing nutation theories for the nonrigid Earth, it is customary to compute the transfer function, i.e., the ratio of the nutation amplitude of the nonrigid Earth to that of a rigid Earth having the same dynamical ellipticity, as a function of the frequency of the forcing torque, and to convolve it with a rigid Earth nutation series. The improvement, during the past decade, of over two orders of magnitude in the accuracy of computation of rigid-Earth nutation series, and the greatly increased precision with which the rate of precession and the nutation coefficients for a large number of frequencies can now be estimated, combine to create the opportunity to seek significant improvements in the theoretical accounting of the observed nutation and precession. Of importance to this effort is the availability of theoretical formulations of the effects of ocean tides (Sasao and Wahr, 1981) and anelasticity (Wahr and Bergen, 1986) on nutations, and the awareness of the potential for important contributions to nutation from electromagnetic couplings of the fluid outer core (FOC) to the mantle (Buffett, 1992) and to the solid inner core (SIC). Enhancement of the existing framework of the analytical theory of nutation and wobble (Mathews et al., 1991) by the inclusion of all the above effects into its basic structure (Mathews et al., 2000) has been a significant step in the effort to construct an accurate nutation series.

We present the new nutation series MHB2000 along with the associated precession rate in the next section, and remark on the quality of its fit to observational data. Salient points of the analytic geophysical theory of nutation and of the process of fitting the theory to observational data will be dealt with in succeeding sections. A brief account of the geophysical implications of the estimates obtained from our fit for various geophysical parameters will form the content of the concluding section.

\section{The new nonrigid-Earth nutation series MHB2000}

The series MHB2000 is given by eq. (7a) below, taken together with the transfer function of eq. (7b). This pair of equations expresses the complex amplitude of any prograde or retrograde circular nutation of the nonrigid Earth in terms of the corresponding rigid-Earth amplitude and the transfer function. For practical 
computations, a resonance formula, given by eq. (10) together with the parameter values listed in Table 1, is provided for the transfer function. The resonance formula reproduces accurately the exact values computed directly from the $\mathrm{dy}$ namical equation (1), except for some frequencies not too far from the free core nutation (FCN) resonance frequency. The inaccuracy at these frequencies arises from the frequency dependence imparted to some of the basic Earth parameters of the theory by ocean tide effects. A correction has to be applied to the amplitude computed from the resonance formula in such cases, so that the result matches the amplitude obtained by solution of the dynamical equation. For this reason, the expression in terms of the resonance formula is supplemented by a table of corrections.

The estimate of 0.0032737875 (with an uncertainty of 5 in the last decimal) obtained for $H_{d}$ is an important ingredient in the computation of the nutation series and must be taken as part of a package along with the series. The rate of general precession corresponding to it is $50288.018 \pm 0.008 \mathrm{mas} / \mathrm{yr}$.

The KSV nutation series of T. A. Herring-an updated version of the empirical IERS1996 series-has provided the closest fit to observations so far. It is instructive to compare the rms of residuals of MHB2000 relative to the observational (VLBI) nutation series with that of KSV. For the VLBI data set covering the period from 1979 to the present, the rms of residuals in $\Delta \psi \sin \epsilon_{0}$ with MHB2000 (KSV) is $0.185(0.189)$ mas, and that in $\Delta \epsilon, 0.187$ (0.194) mas; if only the higher quality data since 1990 are considered, the rms values are considerably lower, at $0.158(0.163)$ mas and $0.158(0.168)$ mas, respectively. In either case, the figures show that MHB2000 provides a somewhat better fit to observations than KSV. What these figures do not show is that in the process of constructing MHB2000, a considerable amount of new and useful information about the Earth's interior is obtained, as will be made manifest in later sections. As for the residuals in individual nutations, the one troublesome one was 0.110 mas (compared to an uncertainty of 0.015 mas) in the prograde annual 18.6year amplitude. Figure 1 shows the time series of residuals for MHB2000 from 1979 to 1999 . The higher quality of the data since 1990 , reflected in the small residuals since then, is evident.

The remainder of this paper will deal with the essentials of the geophysical theory and the data fit on which MHB2000 is based.

\section{Analytic formulation for the dynamical equations}

A forced or free nutation of angular frequency $\tau \Omega_{0}$ or $\tau$ cycles per sidereal day (cpsd), $\Omega_{0}$ being the mean angular velocity of Earth rotation, is necessarily accompanied by a wobble of the Earth's mantle, i.e., a circular motion of its rotation axis around its Tisserand mean axis, with frequency $\sigma$ cpsd as seen from the rotating Earth, where $\sigma=\tau-1$. (The Tisserand mean axis is the principal axis of maximum moment of inertia with tidal deformations ignored.) The amplitude $\tilde{m}(\sigma)$ of this wobble, the amplitudes $\tilde{m}_{f}(\sigma)$ and $\tilde{m}_{s}(\sigma)$ of accompanying wobbles relative to the mantle of the FOC and SIC, and the amplitude $\tilde{n}_{s}(\sigma)$ of the offset of the polar axis of the SIC from that of the mantle, are the dynamical variables of the wobble-nutation problem in the frequency domain. 


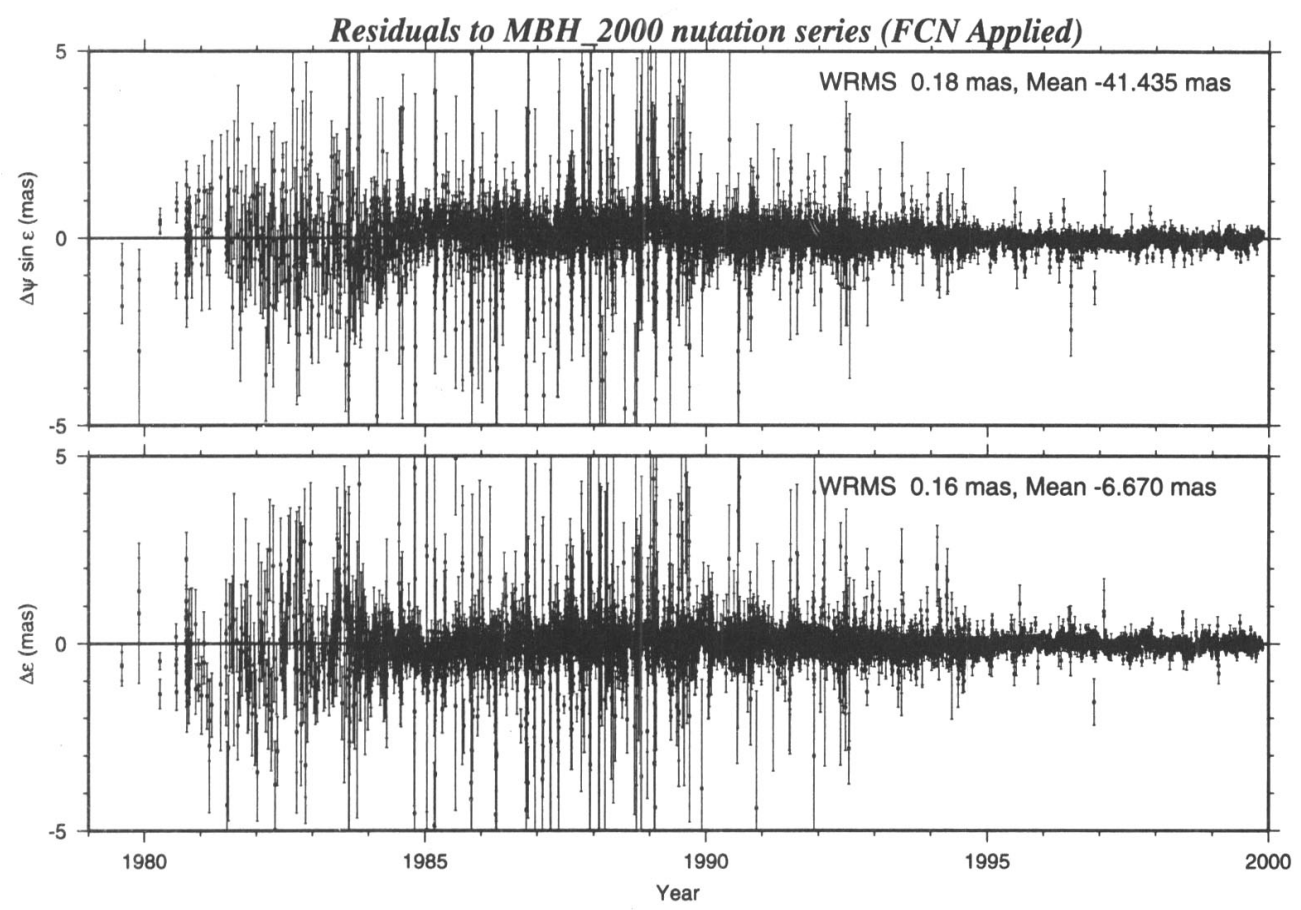

Figure 1. Residuals between the VLBI nutation series and MHB2000. 
Mathews et al. (1991) showed that the four-component column $x(\sigma)$ having these dynamical variables as elements obeys a matrix equation

$$
M(\sigma) x(\sigma)=\tilde{\phi}(\sigma) y(\sigma),
$$

where $\tilde{\phi}(\sigma)$ is the amplitude in nondimensional form of the relevant spectral component of the tide generating potential (TGP), $M$ is the $4 \times 4$ dynamical matrix, and $y$ is a 4-component column. For a free nutation, $\tilde{\phi}=0$. Each element $M_{i j}$ of $M$ as well as each element $y_{i}$ of $y$ is of the form $F+G \sigma$, where the coefficients $F$ and $G$ are simple combinations of the BEP. For an oceanless, elastic, axially symmetric ellipsoidal Earth, the BEP were all real and consisted of the dynamical ellipticities $e, e_{f}, e_{s}$, and the mean equatorial moments of inertia $A, A_{f}, A_{s}$, of the Earth, the FOC, and the SIC, compliance parameters $\kappa, \gamma, \cdots$ representing the deformabilities of the Earth and of its core regions under different types of forcing, the density $\rho_{f}$ of the outer core fluid at the inner core boundary (ICB), and certain other parameters $\left(\alpha_{g}, A^{\prime}, e^{\prime}\right)$ needed to characterize the gravitational coupling between the SIC and the rest of the Earth. The reader is referred to Mathews et al. (1991) for precise definitions, derivations, and other details. Important enchancements were made to the theory by Mathews et al. (2000): inclusion of contributions from mantle anelasticity and ocean tide effects, both complex, in the compliance parameters, and inclusion of electromagnetic couplings of the FOC to the mantle and the SIC due to magnetic fields crossing the core mantle boundary (CMB) and the inner core boundary (ICB).

Eq. (1) is the dynamical equation of the wobble-nutation problem. It contains in succinct form the equatorial components of the equations of angular momentum balance for the whole Earth, the FOC, and the SIC, together with the kinematic equation which relates the instantaneous orientation of the symmetry axis of the SIC to that of its rotation axis.

Given an Earth model such as the Preliminary Reference Earth Model (PREM) of Dziewonski and Anderson (1981), one computes the corresponding hydrostatic equilibrium elliptical structure and evaluates the ellipticity parameters and the moments of inertia; the compliance parameters are computed by solving the deformation equations for the different regions of the Earth under tidal and centrifugal forcing. (Adjustment of the values of some of these basic parameters is done in the process of achieving the "best fit" between theory and observation.) The increments to compliances due to mantle anelasticity are also computable from the deformation equations, given a model for anelastic relaxation. One of a class of two-parameter models used by Wahr and Bergen (1986) is found suitable for the purpose. The ocean tide raised by the same tidal potential that is responsible for the nutation contributes to the tidal deformation of the Earth by its own gravitational action and through crustal loading. Its effect is equivalent to that of incrementing some of the compliance parameters, as one can infer from the theory of Sasao and Wahr (1981). The same theory implies further that the ocean tide contribution to the angular momentum of the Earth may also be incorporated by an appropriate increment to one of the compliance parameters. The increments due to ocean tides are strongly frequency dependent within the diurnal band, in part because of the free core nutation (FCN) resonance in the ocean tides, and partly because of other ocean dynamic factors. Modeling of the frequency dependent ocean tide admittances relevant 
to ocean tidal loading and to the angular momentum of ocean tidal current has to be done before ocean tidal effects can be incorporated into the framework of analytic nutation theory. In Mathews et al. (2000), empirical formulas for the loading and current admittance functions were deduced with the help of the FCN resonance factor for 11 diurnal tides as given by Desai and Wahr (1995) and the ocean loading and current angular momenta for 4 diurnal tides from Chao et al. (1996). The computation of the ocean tide contributions to the various compliances was done using these admittance functions together with loading response parameters computed using the deformation equations.

With incorporation of anelasticity and ocean tide effects thus accomplished, the remaining task was to introduce electromagnetic couplings at the CMB and ICB. The torque between the mantle and the FOC due to the magnetic field at the CMB is proportional to the differential wobble $\tilde{m}$ between the FOC and the mantle, and the torque at the ICB is proportional to $\left(\tilde{m}_{s}-\tilde{m}_{f}\right)$. The effect of these torques is to produce the following modifications in four of the elements of the matrix $M$ :

$$
\begin{gathered}
M_{22} \rightarrow M_{22}+K^{C M B}+K^{I C B} A_{s} / A_{f}, \quad M_{23} \rightarrow M_{23}-K^{I C B} A_{s} / A_{f}, \\
M_{32} \rightarrow M_{32}-K^{I C B}, \quad M_{33} \rightarrow M_{33}+K^{I C B},
\end{gathered}
$$

where the coupling constants $K^{C M B}$ and $K^{I C B}$ are complex. With this, the enhancement of the anlaytical theory to account for anelasticity and ocean tide effects and the electromagnetic couplings is complete.

At this point one has the starting values, corresponding to the Earth model employed, for the BEP appearing in the dynamical equation, including the effects of mantle anelasticity and ocean tides, with the exception of $K^{C M B}$ and $K^{I C B}$. The values of the last two parameters depend on the electrical conductivities of the regions close to the CMB and the ICB and the radial part of the magnetic field at these boundaries. In the absence of adequate information about these properties, one has no a priori values for the two coupling constants; one has to start with trial values.

The next task is to choose from among the BEP an appropriate subset to be varied in order to achieve "best fit" between the nutation amplitudes computed by solution of the dynamical equation (together with the corresponding precession rate) and the observational values estimated by analysis of VLBI data. This question is dealt with in Section 5. Once the choice is made, an iterative least-squares procedure is employed to determine the optimal set of values of the parameters being varied. Finally, with the use of these values in $M$ and $y$, eq. (1) is solved for each frequency of interest to obtain the complex nutation amplitude for each frequency of interest from eqs. (7) below, and the precession rate from eq. (8).

\section{Nutation amplitudes and precession rate from theory}

Given a set of values for the parameters in the dynamical equation, the amplitude $\tilde{m}(\sigma)$ of the wobble produced by any given spectral component of the TGP may be computed from (1) as

$$
\tilde{m}(\sigma)=\left[M^{-1}(\sigma) y(\sigma)\right]_{1} \tilde{\phi}(\sigma)
$$


where the quantity in square brackets is a 4-component column vector, and the subscript 1 indicates the first element of this vector. One may claculate also the precession rate from the ellipticity $e$. For the rigid Earth, the wobble amplitude $\tilde{m}_{R}(\sigma)$ (subscript $R$ for "rigid") reduces to $\tilde{m}_{R}(\sigma)=[e /(e-\sigma)] \tilde{\phi}(\sigma)$.

A nutation with amplitude

$$
\tilde{\eta}(\sigma)=-\tilde{m}(\sigma) /(1+\sigma)
$$

necessarily accompanies a wobble of frequency $\sigma$ cpsd. Eq. (4) is a kinematic relation independent of the Earth's structure and elastic properties. The transfer function $T(\sigma ; e)$ is therefore the same for the wobble and the corresponding nutation:

$$
T(\sigma ; e) \equiv \frac{\tilde{\eta}(\sigma)}{\tilde{\eta}_{R}(\sigma)}=\frac{\tilde{m}(\sigma)}{\tilde{m}_{R}(\sigma)}=\frac{e-\sigma}{e}\left[M^{-1}(\sigma) y(\sigma)\right]_{1} .
$$

For an oceanless elastic Earth, Mathews et al. (1991) obtained a resonance formula for $T(\sigma ; e)$ in the general form

$$
T(\sigma ; e)=R+(1+\sigma) R^{\prime}+\sum_{\alpha=1}^{4} \frac{R_{\alpha}}{\sigma-\sigma_{\alpha}},
$$

subject to certain sum rules relating the coefficients. Since the ellipticity $e_{R}$ assumed in computing the rigid-Earth amplitudes need not be exactly equal to $e$ of the real Earth-indeed, $e_{R}$ itself differs from one rigid Earth theory to another-it is necessary to introduce a generalized transfer function $T\left(\sigma ; e \mid e_{R}\right)$ :

$$
\tilde{\eta}(\sigma ; e)=T\left(\sigma ; e \mid e_{R}\right) \tilde{\eta}_{R}\left(\sigma ; e_{R}\right) .
$$

(The ellipticity is shown explicitly here as an extra argument for $\tilde{\eta}$ and $\tilde{\eta}_{R}$.) This transfer function has the expression

$$
T\left(\sigma ; e \mid e_{R}\right)=\left(1-\sigma / e_{R}\right)\left[M^{-1}(\sigma) y(\sigma)\right]_{1} .
$$

Computation of the nutation amplitudes $\tilde{\eta}(\sigma ; e)$ is done by determining the transfer function of $(7 \mathrm{~b})$ by solving the dynamical equation, and then using (7a).

The rigid-Earth nutation amplitudes needed for the second step were taken, in Mathews et al. (2000), from the series REN-2000 of Souchay et al. (1999). The computations need to be repeated with RDAN of Roosbeek and Dehant (1998) and SMART of Bretagnon et al. (1998), once these series are implemented in the software used for estimation of nutation amplitudes from VLBI data.

It is known (Fukushima, 1991) that general relativistic effects give rise to the so-called geodesic nutation contributions. The significant contributions are -0.0304 mas and +0.0304 mas, respectively, to the prograde and retrograde annual nutations, and -0.0004 mas and +0.0004 mas to the semiannual ones. The atmospheric (thermal $S_{1}$ tide) contribution to the prograde annual nutation amplitude as estimated from the post-fit residuals was -0.010 mas in phase and 0.120 mas out of phase. In Mathews et al. (2000), both the geodesic nutation and atmospheric contributions were added to the relevant amplitudes computed from eqs. (7). 
As for the precession rate $P$, the correction $\Delta P \equiv\left(P-P_{I A U}\right)$ to its IAU value is related to the deviation of $H_{d}$ from the assumed rigid-Earth value $H_{d R}$ by

$$
\Delta P=\left(P_{R}-P_{I A U}\right)+\left(d P_{R} / d H_{d R}\right)\left(H_{d}-H_{d R}\right) .
$$

The value of $d P_{R} / d H_{d R}$ as computed by Bretagnon (private communication, $1999)$ was used by Mathews et al. (2000): $d P_{R} / d H_{d R}=15395304.60763 \mathrm{mas} / \mathrm{yr}$. Eq. (8) provides the needed theoretical relation between $\Delta P$ and $e$, since $H_{d}=$ $e /(1+e)$.

\section{Least-squares fit of theory to data}

To make a least-squares fit of the theory outlined above to nutation data, one needs first to identify those of the BEP which have dominant roles in determining the nutation amplitudes $\tilde{\eta}(\sigma)$. It turns out that apart from $e$, the most important parameter of all, the other BEP which significantly influence nutations enter through the following six combinations:

$$
\begin{gathered}
p_{1}=\left(A / A_{m}\right)(e-\kappa), \\
p_{2}=\left(A_{f} / A_{m}\right)(e-\gamma), \\
p_{3}=\left(A / A_{m}\right)\left(e_{f}-\beta+\operatorname{Re} K^{C M B}+\operatorname{Re} K^{I C B} A_{s} / A_{f}\right), \\
p_{4}=\alpha_{2} e_{s}+\nu-\operatorname{Re} K^{I C B}, \\
p_{5}=\left(A / A_{m}\right)\left(\operatorname{Im} K^{C M B}+\operatorname{Im} K^{I C B} A_{s} / A_{f}\right), \\
p_{6}=\operatorname{Im} K^{I C B} .
\end{gathered}
$$

This observation led to the choice of $e$, along with the following six independent parameters through which the $p_{i}$ can be varied, for adjustment in the least-squares fitting process: the ellipticity $e_{f}$ of the fluid core, the compliance parameters $\kappa$ and $\gamma$ representing the deformabilities of the whole Earth and the FOC, respectively, under tidal forcing, $\operatorname{Im} K^{C M B}$, $\operatorname{Re} K^{I C B}$, and $\operatorname{Im} K^{I C B}$.

The input data which are to be fitted by varying the above parameters consists of estimates obtained from VLBI data analysis for the precession rate and the amplitudes, in-phase and out-of-phase, of a number of circular nutations, taken in prograde-retrograde pairs. The data include the variance-covariance matrix of these estimates. Mathews et al. (2000) carried out independent fits using the estimates obtained from analyses of GSFC and USNO VLBI data sets covering the period 1979-1999, using 85 observables for the fit: the in and out of phase parts of the amplitudes of 42 nutations (in 21 pairs), along with the rate of precession in longitude. The set of estimates obtained for the seven BEP from the least-squares fits were essentially independent of the data sets used for the inputs: there were no statistically significant differences.

The estimation process itself was one of simultaneous least-squares fitting of the theoretical expressions for $\Delta P$ and the 42 complex $\tilde{\eta}(\sigma)$ to the input data set, taking into account the standard deviations and correlations embodied in the variance-covariance matrix forming part of the data set. The details of the nonlinear least-squares procedure, which involves an iterative process with updating of the values of the BEP and recomputation of the partials at each stage, may be found in Mathews et al. (2000). 


\section{Resonance formulas}

For the computation of the nonrigid-Earth nutation series, it is far more convenient to use a resonance formula for the generalized transfer function than to compute $(7 \mathrm{~b})$ by solving the dynamical equation for each term of the series. If the BEP were frequency independent, one could simply compute the eigenvalues $\sigma_{\alpha},(\alpha=1, \cdots, 4)$, and the corresponding eigenvectors of the problem by solving eq. (1) with $\tilde{\phi}(\sigma)$ set equal to zero, and compute therefrom the coefficients in the resonance expansion

$$
T\left(\sigma ; e \mid e_{R}\right)=\frac{e_{R}-\sigma}{e_{R}+1} N_{0}\left(1+(1+\sigma) \sum_{\alpha=1}^{4} \frac{N_{\alpha}}{\sigma-\sigma_{\alpha}}\right) .
$$

In the present context, however, the strong variation of the ocean tide admittances across the diurnal tidal band imparts to the compliance parameters a significant dependence on the frequency of the wobble. So the eigenvalues $\sigma_{\alpha}$ and eigenvectors obtainable from eq. (1) and the parameters $N_{\alpha}$ evaluated using them, are all dependent on the wobble frequency for which the compliances are evaluated. By evaluating the $\sigma_{\alpha}$ and $N_{\alpha}$ corresponding to a wide range of diurnal wobble frequencies, extracting, for each of them, a part that varies linearly with $\sigma$, and inserting these linearly varying parameters instead of the constants into (9) and rearranging terms, Mathews et al. (2000) obtained the following modified form for the resonance formula:

$$
T\left(\sigma ; e \mid e_{R}\right)=\frac{e_{R}-\sigma}{e_{R}+1}\left[N_{0}+(1+\sigma)\left(Q_{0}+\sum_{\alpha=1}^{4} \frac{Q_{\alpha}}{\sigma-s_{\alpha}}\right)\right] .
$$

The values of the "effective" resonance frequencies $s_{\alpha}$ and of the other parameters in this formula are listed in Table 1.

This "modified resonance formula" (MRF) is still not expected to give full agreement with the exact results from direct solution of the dynamical equation for nutations that are not too far from the FCN resonance or are of large amplitude. When a nutation amplitude computed from eq. (7a) taken together with the MRF (10) differs from the result of the direct evaluation using (7a) and (7b), the difference between the two has to be applied to the former as a correction. A table of corrections is therefore used along with the MRF. The only corrections exceeding $4 \mu$ as are $(-86,111),(-6,9),(-22,28)(1,-15) \mu$ as (in phase, out of phase) respectively, to the retrograde annual, retrograde and prograde half-yearly, and prograde fortnightly, nutations. For the full set of corrections rounding off to $1 \mu$ as or more, see Mathews et al. (2000). Finally, the geodesic nutation and atmospheric contributions referred to earlier are to be added. 
Table 1. MHB2000: Parameters for use in the resonance formula (10)

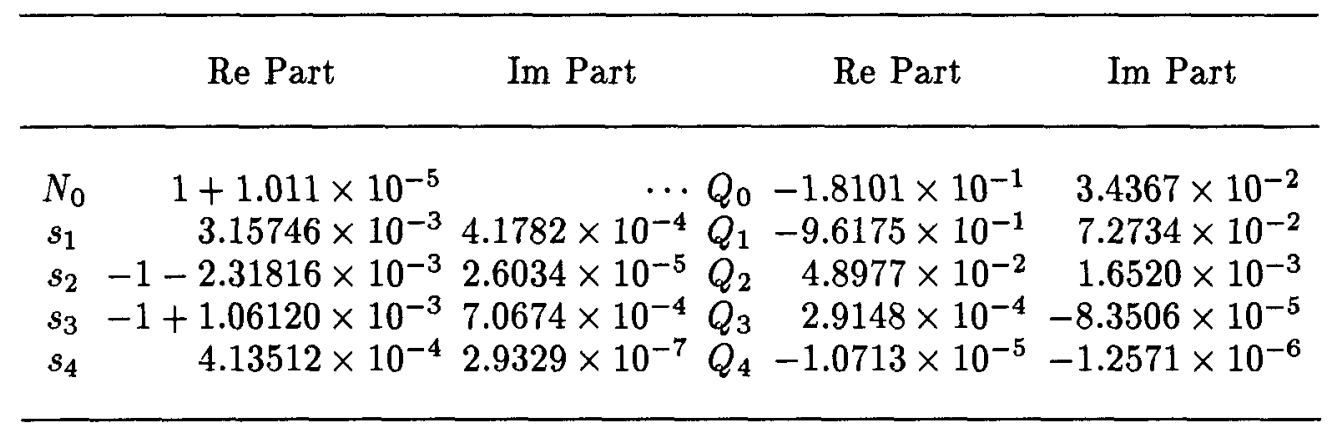

\section{Discussion and concluding remarks}

Apart from the level of agreement that has been achieved between theory, as given by MHB2000, and observations of nutation and precession, information gained about the Earth's interior through the estimates obtained for Earth parameters is in itself of great interest. For instance, the very possibility of estimating $K^{I C B}$ opens up a means of learning something about the magnetic field at the inner core boundary - a field that is not detectable from the outside because of shielding by the highly conducting outer core. The estimate found for $K^{I C B}$ suggests an rms magnetic field of over 65 gauss at the ICB, though the estimate could change somewhat if the enviornment of the ICB were varied from that given by the Earth model. Similarly, the estimate for $\operatorname{Im} K^{C M B}$ calls for the presence of at least a $0.2 \mathrm{~km}$ layer at the bottom of the mantle, with conductivity close to that of the core fluid, and an rms radial magnetic field of at least 7 gauss at the CMB. Another finding is that of only $3.8 \%$ excess (nonhydrostatic) ellipticity for the fluid core, as against previous estimates of nearly $5 \%$. Such findings place new constraints on theories of the geodynamo, mantle convection, chemistry of the lowermost layers of the mantle, etc. It is also noteworthy that the estimate of 970 days, with an uncertainty of only $5 \%$, that has been found for the period of the inner-core-induced normal mode predicted by theory (called PFCN or FICN) indicates positive detection of this mode for the first time. The period found for the familiar FCN mode is $430.2 \pm 0.2$ days. A seemingly unrealistic period of $384.2 \pm 0.7$ days found for the Chandler resonance period proved most interesting: when corrected for the difference in anelasticity and ocean tide contributions to compliances between the diurnal frequency of tidal forcing and the low frequency of the free Chandler mode, the eigenperiod turned out to be $432.2 \pm 0.8$ days, just about what is actually observed.

\section{References}

Bretagnon, P., Francou, G., Rocher, P., and Simon, J.-L., 1998, Astron. Astrophys., 329, 329-338.

Buffett, B. A., 1992, J. Geophys. Res., 97, 19581-19597.

Chao, B. F., Ray, R. D., Gipson, J. M., Egbert, G. D., and Ma, C., 1996, J. Geophys. Res., 101, 20151-20163. 
Dehant, V., Proc. IAU Colloq. 180 (This volume).

Dehant, V. and Defraigne, P., 1997, J. Geophys. Res., 102, 27659-27688.

Desai, S. and Wahr, J. M., 1995, J. Geophys. Res., 100, 25205-25228.

Dziewonski, A. M. and Anderson, D. L., 1981, Phys. Earth Planet. Inter., 25, 297-356.

Fukushima, T., 1991, Astron. Astrophys., 244, L11-L12.

Mathews, P. M., Herring, T. A., and Buffett, B. A., 2000, (Submitted to J. Geophys. Res.)

Mathews, P. M., Buffett, B. A., Herring, T. A., and Shapiro, I. I., 1991, J. Geophys. Res., 96, 8219-8242.

McCarthy, D. D., IERS Conventions, 1996, IERS Technical Note No. 21.

Roosbeek, F. and Dehant, V., 1998; Celestial Mech., 70, 215-253.

Sasao, T. and Wahr, J. M., 1981, Geophys. J. R. Astron. Soc., 64, 729-746.

Shirai, T. and Fukushima, T., 2000, Proc. IAU Colloq. 180 (This volume).

Souchay, J., Loysel, B. Kinoshita, H., and Folgueira, M., 1999, Astron. Astrophys. Suppl. Ser., 135, 111-131.

Wahr, J. M., 1981, Geophys. J. R. Astron. Soc., 64, 705-727.

Wahr, J. and Bergen, Z., 1986, Geophys. J. R. Astron. Soc., 87, 633-668. 